\title{
ARMY MORBIDITY RECORDS NEED OR NUISANCE?
}

\author{
LT COL R H HARDIE, OBE, MB, ChB, RAMC \\ Royal Army Medical College \\ Donald R COID, B Med Sc, BM, BS, MRCP \\ London School of Hygiene and Tropical Medicine
}

SUMMARY: Records of in-patients morbidity should serve a number of important functions in the army; these include providing statistics for research and planning. However there has been some suggestion that the two 'information systems' which convey this data are ineffectual. This hypothesis was explored by examination of statistics gathered for the years 1969 to 1977. Deficiences in both information systems were discovered. It is suggested that there should be some revision of the methods of collecting morbidity statistics.

\section{Introduction}

The Army Medical Services are faced with the problem of providing a comprehensive system of health care under resource restraints. This demands efficient use of facilities, one of which is the system of collecting and recording morbidity statistics. The stated objectives of morbidity information are:-

a. To record details of diseases and treatment for immediate and future use.

b. To indicate the probable case loads in hospitals and hospital departments.

c. To provide material for medical research and statistics.

d. To provide information to the responsible authorities dealing with pension claims and for the investigation of complaints and enquiries which may arise.

There are two systems of collection of in-patient data. One system uses the form $\mathrm{F}$ Med 14 to report discharge data. The other system uses the form AF W3166 to report the numbers of admissions by diagnosis.

The F Med 14 system records full patient details including discharge diagnosis. These details are stored by computerisation. This system is 'record linked' but has the disadvantage that delay tends to occur in accumulating the information. It may be that unusual occurrences are well reported in comparison to the more common illnesses.

The AF W3166 system records numerically by diagnosis all admissions to medical establishments with in-patient facilities. The completed AF W3166 for each district and command is normally received by Stats (G) 4 by the middle of the month following that to which it relates. The AF W3166 form was designed with an emphasis on infectious diseases. Furthermore the disease groupings are currently coded according to the International Classification of Diseases (ICD) 8th revision, whereas F Med 14 has used the 9th revision since 1979.

This paper describes some of the results of an investigation into the effecttiveness of the F Med 14 and AF W3166 systems. 


\section{Methods}

The total number of admissions recorded (AF W3166) together with deaths and discharges (F Med 14) for the years 1969 to 1977 have been tabulated (Table I). The ratio of F Med 14 returns to AF W3166 numbers is illustrated graphically (Fig 1).

\section{Table I}

British Army males recorded admissions (AF W3166) and death and discharges (F Med 14) for 1969 to 1977

\begin{tabular}{ccc}
\hline Year & $\begin{array}{c}\text { AF W3166 recorded } \\
\text { admissions (number) }\end{array}$ & $\begin{array}{c}\text { F Med 14 death and } \\
\text { discharges (number) }\end{array}$ \\
\hline 1969 & 40,441 & 36,260 \\
1970 & 35,996 & 29,897 \\
1971 & 35,274 & 28,221 \\
1972 & 37,183 & 28,897 \\
1973 & 34,418 & 26,763 \\
1974 & 32,352 & 25,479 \\
1975 & 33,403 & 25,053 \\
1976 & 34,020 & 25,064 \\
1977 & 31,625 & 22,598 \\
\hline
\end{tabular}

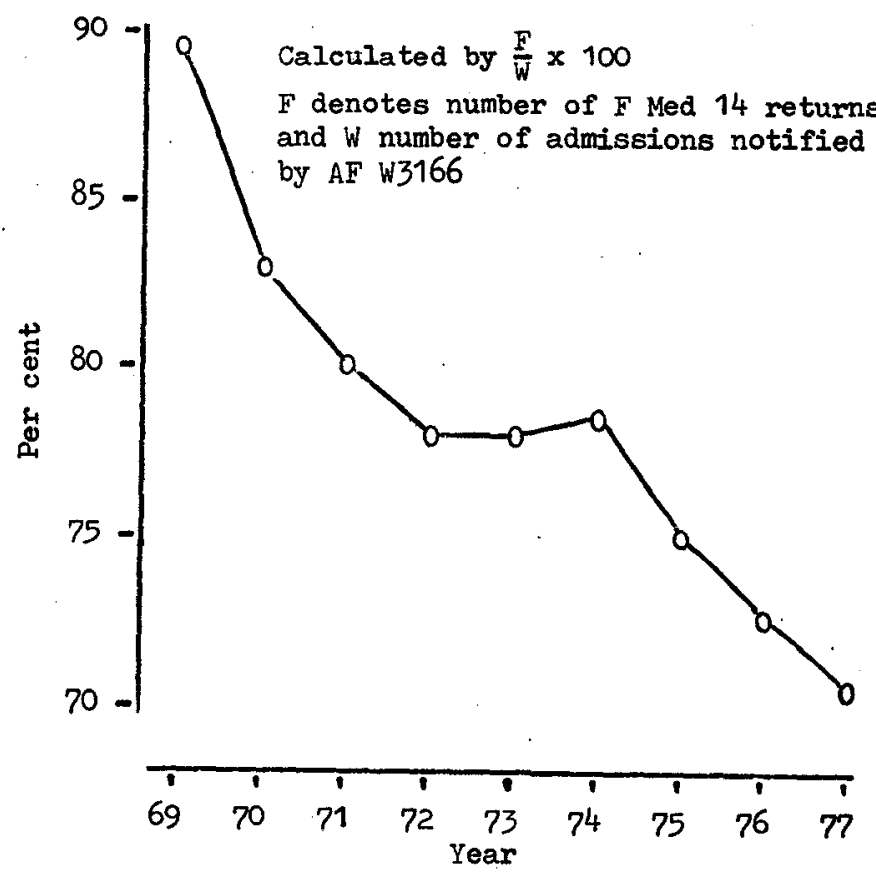

Fig. 1 Ratio of F Med 14 returns to AF W3166 notifications. 
For the F Med 14 system, the numbers of deaths and discharges of British Army males for the years 1969 to 1977 were obtained. These are summated according to Chapter of the ICD (8th revision). Similarly recorded were the numbers of admissions according to AF W3166. The two sets of data are compared (Table II).

For the F Med 14 system the number of patients falling into each threedigit category of the ICD were examined. Diseases are recorded for which there is an average of more than 150 discharges per year, but for which there is no request for specific information on the AF W3166 form.

\section{Results}

Table I - Number of recorded admissions (AF W3166) and deaths and discharges ( $F$ Med 14) of British Army males for the period 1969 to 1977.

Table II - Number of in-patients of British Army males by ICD Chapter. AF W3166 and F Med 14 data compared for the period 1969 to 1977.

Table III - British Army male discharges and deaths for the period 1969 to 1977. Three digit ICD diagnoses for which there averaged more than 150 discharges and deaths per year and are currently reported by AF W3166 under 'All other diseases'.

Figure 1 - Ratio of F Med 14 returns to AF W3166 notifications for British Army males for the period 1969 to 1977.

Table II

British Army males in-patients by ICD chapter, AF W3166 and F Med 14 data compared for 1969 to 1977

\begin{tabular}{llcc}
\hline ICD chapter & \multicolumn{1}{c}{ Disease group } & $\begin{array}{c}\text { Number of patients } \\
\text { AF }\end{array}$ W3166 data & F Med 14 data \\
\hline I & Infectious and parasitic & 18,483 & 16,008 \\
II & Neoplasm & 1,565 & 2,674 \\
III & Endocrine, nutritional and metabolic & 31 & 2,159 \\
IV & Blood diseases & $*$ & 608 \\
V & Mental disorders & 7,993 & 6,808 \\
VI & Nervous disorders & 3,720 & 5,653 \\
VII & Circulatory diseases & 4,078 & 7,349 \\
VIII & Respiratory diseases & 42,949 & 46,269 \\
IX & Diseases of digestive system & 12,178 & 32,775 \\
X & Genito-urinary diseases & $*$ & 7,657 \\
XII & Skin diseases & 9,058 & 13,034 \\
XIII & Musculoskeletal diseases & 7,428 & 20,636 \\
XIV & Congenital abnormalities & $*$ & 1,209 \\
XVI & Symptomatic & 1,857 & 18,827 \\
& 'All other diseases' & 134,658 & Not applicable \\
& Accidents, poisoning, violence & 71,010 & 68,330 \\
\hline Totals & & 315,008 & 249,696 \\
\hline
\end{tabular}

*Information not requested for all diseases in these chapters by AF W3166 and therefore numbers included in 'all other diseases' 
Table III

British Army male discharges and deaths for the period 1969 to 1977. Three digit ICD diagnoses for which there averaged more than 150 discharges and deaths per year are currently reported on AF W3166 under 'All other diseases'

\begin{tabular}{llr}
\hline 8th ICD number & \multicolumn{1}{c}{ Description/diagnosis } & $\begin{array}{c}\text { Average number } \\
\text { discharges and } \\
\text { deaths } \\
\text { (1969 to 1977) }\end{array}$ \\
\hline 465 & Acute urinary tract infection & 1418 \\
504 & Deflected nasal septumi & 255 \\
520 & Disorders of tooth development & 372 \\
521 & Disorders of hard tissues of teeth & 488 \\
522 & Disorders of pulp and periapical tissue & 301 \\
525 & Other disorders of teeth & 387 \\
535 & Gastritis/duodenitis & 201 \\
536 & Disorder of function of stomach & 152 \\
605 & Redundant prepuce & 182 \\
725 & Displacement intervertabral disc & 249 \\
729 & Other diseases of joints & 324 \\
731 & Synovitis/bursitis/tenosynovitis & 157 \\
733 & Other diseases of muscle & 219 \\
782 & Cardiovascular symptoms & 150 \\
783 & Respiratory symptoms & 185 \\
785 & Abdominal symptoms & 701 \\
786 & Genito-urinary symptoms & 156 \\
793 & Observation & 159 \\
\hline
\end{tabular}

\section{Discussion}

The declining number of British Army male admissions between the years 1969 to 1977 appears to have been accompanied by a drop in the proportion of admissions for which F Med 14 is completed. There are serious discrepancies between numbers reported for each ICD Chapter by the two different systems of morbidity recording. About 40 per cent of AF W3166 numbers are reported under 'All other diseases' category; the information specifically requested in the AF W3166 form does not telegraph information regarding several illnesses affecting large numbers of Army personnel.

\section{Acknowledgements}

The authors gratefully acknowledge the help and encouragement given by Brig D E Worsley, L/RAMC, Lt Col I P Crawford, RAMC, Dr J S A Ashley, London School of Hygiene and Tropical Medicine and Mr B J Oelman, MOD, Stats (G) 4, Stanmore. 